\title{
Tabularia
}

\section{La diaspora normande vue par les marges : la principauté d'Antioche entre histoire et historiographie}

The norman Diaspora viewed from the margins: the principality of Antioch between history and historiography

La dispora normanna vista dai margini: il principato di Antiochia tra storia e storiografia

\section{Luigi Russo}

\section{OpenEdition}

\section{Journals}

Édition électronique

URL : http://journals.openedition.org/tabularia/2619

DOI : $10.4000 /$ tabularia.2619

ISSN : 1630-7364

Éditeur :

CRAHAM - Centre Michel de Boüard, Presses universitaires de Caen

Référence électronique

Luigi Russo, «La diaspora normande vue par les marges : la principauté d'Antioche entre histoire et historiographie », Tabularia [En ligne], Mémoires normandes d'Italie et d'Orient, mis en ligne le 08 septembre 2016, consulté le 30 avril 2019. URL : http://journals.openedition.org/tabularia/2619; DOI : 10.4000/tabularia.2619 


\title{
La diaspora normande vue par les marges: la principauté d'Antioche entre histoire et historiographie
}

\author{
The norman Diaspora viewed from the margins: \\ the principality of Antioch \\ between history and historiography
}

\section{La dispora normanna vista dai margini: il principato di Antiochia tra storia e storiografia}

\author{
Luigi Russo \\ Università Europea di Roma \\ luigi.russo@unier.it
}

\section{Résumé:}

La contribution a l'intention d'analyser la manière dont a été caractérisée l'expansion normande dans l'Orient latin dans les décennies suivant la première croisade. Une attention particulière sera accordée aux événements de l'établissement de groupes familiaux ou individus d'origine normande dans la principauté d'Antioche, fondée par Bohémond de Hauteville en 1098. La rencontre entre les chevaliers occidentaux arrivés à la suite de la croisade et les populations locales a été interprétée de façon très variable par l'historiographie de l'époque moderne, qui s'est souvent appuyée sur un concept d'identité religieuse peu adapté aux sources médiévales. Les Normands qui ont dominé la scène politique de la principauté d'Antioche au cours des trois premières décennies du XII siècle ont été confrontés à une population majoritaire avec laquelle ils ont dû établir des relations de collaboration. Cependant, la documentation à notre disposition donne l'image d'un monde belliqueux, radicalement opposé à la fois à un monde islamique fragmenté et à l'Empire byzantin.

Mots-clés: Antioche, Première croisade, Bohémond de Hauteville, Orient latin, Normanitas, Historiographie

\begin{abstract}
:
The contribution aims to analyze the ways that characterized the Norman expansion in the Latin East in the decades after the First Crusade. Particular attention will be given to the events of the settlement of family groups or individuals of Norman origin in the principality of Antioch, founded by Bohemond of Hauteville in 1098. The confrontation between the Western crusaders and the local population has been interpreted in a very different way from the modern historiography that has applied categories borrowed from concepts of religious identity poorly applicable to medieval sources. The Normans who dominated the political scene of the principality of Antioch in the first decades of the twelfth century were faced with a majority which had to build collaborative relationships. However, just the documentary framework at our disposal tends to convey the image of a warlike world, radically opposed to the fragmented Islamic world and the Byzantine Empire.
\end{abstract}

Keywords: Antioch, First Crusade, Bohemond of Hauteville, Latin East, Normanitas, historiography 
Riassunto:

Il contributo intende analizzare le modalità che caratterizzarono l'espansione normanna nell'Oriente latino nei decenni successivi la prima crociata. Particolare attenzione sarà data alle vicende del popolamento di gruppi familiari o individui singoli di origine normanna nel principato di Antiochia fondato da Boemondo d'Altavilla nel 1098. L'incontro tra i cavalieri occidentali giunti al seguito della crociata e le popolazioni locali è stato interpretato in maniera molto variabile dalla storiografia d'età moderna che ha sovente adottato categorie di pensiero debitrici di un concetto di identità religiosa scarsamente applicabile alle fonti medievali. I Normanni che dominarono la scena politica del principato di Antiochia nel primo trentennio del XII secolo si trovarono di fronte una popolazione maggioritaria con cui dovettero costruire rapporti di collaborazione. Tuttavia, proprio il quadro documentario a nostra disposizione tende a trasmettere l'immagine di un mondo bellicoso, radicalmente opposto sia al frammentato mondo islamico che all'Impero bizantino.

Parole chiave: Antiochia, Prima crociata, Boemondo d'Altavilla, Oriente latino, Normanitas, storiografia

Ce n'est pas ici le lieu pour discuter de manière exhaustive de la succession des événements qui conduisit à l'expansion normande pendant le XII siècle en Orient; la présence d'un grand nombre d'œuvres importantes fournissant une documentation presque complète des événements survenus au cours de la première croisade, qui ont abouti à la naissance de la principauté d'Antioche, nous permet d'éviter de nous attarder trop longuement sur le projet politique obstinément poursuivi par Bohémond de Hauteville ${ }^{1}$. Je voudrais plutôt ici vous proposer quelques réflexions touchant le domaine des études normandes en général, en m'appuyant uniquement sur le cas de la principauté d'Antioche au cours des trois premières décennies du XII siècle, durant lesquelles le leadership était sous le contrôle des héritiers des Hauteville. Ces héritiers continuèrent à conserver la mémoire des exploits du Magnus Boamundus, comme en témoigne un document publié en juillet 1134 par la princesse Alice, épouse du défunt Bohémond II, qui reprenait d'ailleurs le formulaire de chancellerie dans une forte continuité avec la documentation du mari ${ }^{2}$. Les événements historiques qui ont conduit à la destruction de l'Outremer expliquent en effet l'extrême pauvreté

1. Sur le chef normand voir FLORI, 2007; RUSSO, 2009; LORENZ, 2007. Sur les États latins d'Orient, voir BARber, 2012 et Tyerman, 2012, p. 171 sq.; sur Antioche, Asbridge, 2000; ID., 1999, p. 305 325: CAHEN, 1940, est toujours une œuvre de référence, en particulier les p. 205-345, mais aussi ReY, 1896, p. 321-407; ID., 1900-1901, p. 116-157. En dernier lieu, EdBury, 2013, p. 241-248.

2. Cf. MAYer, 1993, doc. 2, p. 113: «ego Adelicia Balduini regis Hierosolymitani Latinorum secondi filia, uxor quondam domini Boamundi, Magni Boamundi filii [souligné par l'auteur], excellentissimi Antiochenorum principis, dei gratia principissa Antiochena... ». Voir également Chartes de l'abbaye..., éd. C. KoHLER, 1899, doc. XX, p. 129-130. On trouvera un commentaire dans Asbridge, 2003, p. 29-47, à la p. 40. Voir la même intitulatio rédigée avec le nom de Bohémond II «ego Boamundus magni Boamundi filius» dans le privilège en faveur des Hospitaliers de mars 1127 : cf. HIESTAND, 1997, p. 27-46, à la p. 45; et dans le privilège en faveur des Génois de décembre 1127: cf. Codice Diplomatico..., éd. C. Imperiale di Sant'Angelo, 1936, p. 57-59, doc. 47. Enfin, voir la donation dans les années 1137-1138 au Saint Sépulchre du clerc Riso, dans laquelle il se nomme Boamundi Antiocheni principis magnique Boamundi filii quondam cappellanus: Le Cartulaire du chapitre..., éd. G. BresC-BAUTIER, 1984, doc. 75, p. 174. 
de la documentation en notre possession pour Antioche ${ }^{3}$ : seuls les témoignages historiographiques semblent avoir été sauvés d'une façon appréciable (mais pas tous $)^{4}$, tandis que la conservation des documents d'archives est souvent liée à des événements occasionnels ou fortuits, comme dans le cas d'un document rédigé en arabe en 1213, qui témoigne de la relation entre les communautés chrétiennes d'Antioche de langue grecque et latine, par bonheur conservé dans les archives siciliennes ${ }^{5}$. Il s'agit de documents qui peuvent - à notre avis - malgré leur caractère "atypique " ${ }^{6}$, fournir d'importantes indications sur l'expansion normande.

Le cas présenté ici correspond à la dernière extension de la diaspora qui conduisit les hommes du Nord à devenir les protagonistes de l'Europe durant l'époque médiévale, en une série de vicissitudes sur lesquelles de nombreux chercheurs se sont longtemps penchés pour tenter d'analyser les faits et de trouver un trait d'union entre des événements situés dans des contextes radicalement différents, comme ceux concernant le Sud de l'Italie, la Terre Sainte, l'Angleterre et la Normandie ${ }^{7}$. Ces efforts, cependant, se sont fondés sur la foi d'une documentation extrêmement abondante pour l'Angleterre et utile pour le médiéviste qui travaille sur des sources des $\mathrm{XI}^{\mathrm{e}}$ et $\mathrm{XII}^{\mathrm{e}}$ siècles, alors que pour Antioche, au contraire ${ }^{8}$, nous sommes confrontés à l'extrême raréfaction des documents à notre disposition. Une différence dont on devra tenir compte au moment d'exprimer un jugement général sur les caractéristiques de la «diaspora normande », expression brillante récemment utilisée par David Bates pour définir le mouvement d'expansion du monde normand ${ }^{9}$.

\section{Le départ de Bohémond}

Nous abordons donc le cas d'Antioche sur la base des documents en notre possession. On situe dans les mois qui ont suivi la défaite de Harran (1104) le discours lucide tenu par Bohémond dans la cathédrale Saint-Pierre d'Antioche

3. Pour les sources sur le comté d'Édesse, voir Amouroux-Mourad, 1988, p. 5-8.

4. Sur cette question, voir MAYer, 1993, p. 75-122, à la p. 75, et aussi MurRAY, 1997, p. 349-359, et la discussion de Figliuolo, 1995, p. 375-379. On trouvera une analyse exhaustive des sources dans Asbridge, 2000, p. 5-13. Edbury, Rowe, 1988, p. 46, considèrent que Guillaume de Tyr a consulté «a history of Antioch for the second quarter of the century which provided information on the dynastic problems in the principality following the death of Bohemond II in 1130 [...]». Cette source a aujourd'hui disparu.

5. Voir CAHen, 1971, p. 285-292.

6. Nous renvoyons à l'opinion - sans doute réductrice - de Figliuolo, 1995, p. 375, sur Raoul de Caen ( a carattere quasi esclusivamente encomiastico») et Albert d'Aix..., éd. S.B. Edgington, 2007.

7. Brown, 2010 [2 éd. orig. Woodbridge 1984], p. 157-201; Matthew, 1987; Chibnall, 2005, p. 108-118.

8. Pour les sources, voir Domesday Book..., éd. A. Williams - G.H. Martin, 2002; The great roll..., éd. J.A. Green, 2012; Richard Fitz Nigel..., éd. C. Johnson, 1983. Voir aussi Records..., éd. N. Vincent, 2009; Huscroft, 2009; Thomas, 2008; Clanchy, 200633 Carpenter, 2003; Clanchy, 2012 ${ }^{3}$; Chibnall, 1993² ; Green, 1986.

9. L'ouvrage de David Bates (BATES, 2013), est fondamental: voir, en particulier, p. 1-27 et p. 178-185; voir aussi STRINGER et JOTISCHKy (éd.), 2013, p. 1-8. On trouvera une excellente mise à jour dans BAtes et BAUduin, 2016. 
devant ses partisans, y compris son cousin Tancrède ${ }^{10}$ spécialement convoqué à Édesse, où il avait été appelé pour résoudre une délicate question de régence dans la région qui lui avait été confiée après la capture du comte Baudouin dit «de Bourcq" (le futur roi Baudouin II de Jérusalem) ${ }^{11}$.

«O grands, dans les circonstances où nous nous trouvons, il nous faut user d'une grande prudence, sans quoi nous allons périr. Les Gentils ont prévalu contre nous: les Grecs et les Turcs nous ferment tous les chemins. Nous avons irrité les deux puissances les plus opulentes du monde, Constantinople et la Perse; l'Orient nous assiège par terre, l'Occident par terre et par mer. En effet, sans parler de beaucoup d'autres villes, Artah, qui fut jusqu'à présent le bouclier d'Antioche, Artah maintenant tend ses arcs et aiguise ses flèches contre nous ${ }^{12}$. Nous sommes en petit nombre, et cependant nous devenons de jour en jour moins nombreux; nos forces en outre ont été infiniment diminuées par la perte d'un seul homme, le comte d'Édesse. Ainsi donc soyez vigilants, examinez très attentivement ce qu'il convient de faire en des circonstances aussi graves. Moi je vous dirai en peu de mots ce que je pense. Il nous faut aller chercher des forces nouvelles au-delà des mers; il nous faut soulever en notre faveur les peuples des Gaules (= Galliarum populi concitandi): c'est par cette décision que nous serons sauvés, et par aucune autre ${ }^{13}$.

Pour interpréter ce discours, extraordinaire pour la lucidité avec laquelle est illustrée la réalité de ces années difficiles, il convient de rappeler que, bien que construit d'après des témoignages directs ${ }^{14}$, il a été reformulé sur la foi d'événements successifs présentés dans la campagne de propagande anti-byzantine orchestrée par Bohémond en Occident ${ }^{15}$ si bien qu'il doit être évalué avec la

10. Sur la question, voir Russo, 2011, p. 169 et note 25 [réimprimé dans ID., 2014, p. 23-36].

11. Voir Anonymi auctoris chronicon..., trad. AbounA, 1974, chap. 263, p. 51-52 [= texte original: Anonymi auctoris chronicon..., éd. СНАвот]. Sur les événements, il faut voir aussi Kemal ed-Din..., in Recueil des historiens des croisades, 1884, p. 592-593.

12. La chute d'Artah est mentionnée dans Radulphus Cadomensis..., éd. E. D’Angelo, 2011, par. 434, p. 126, 11. 4325-4330, mais elle eut lieu au printemps de 1105, c'est-à-dire après le départ de Bohémond. On trouvera plusieurs détails dans Kemal ed-Din..., in Recueil des historiens des croisades, 1884, p. 593, et dans The Damascus Chronicle..., éd. H.A.R. GiBB, 2002, année 498, p. 69-70.

13. Radulphus Cadomensis..., éd. E. D’Angelo, 2011, chap. 436-437, p. 127, 11. 4347-4360: «Magna opus est, o proceres, hac in tempestate prouidentia: quam si negligimus, perimus! Invaluit contra nos gentilitas, uias nobis circumcirca obstruxuerunt Greci et Turci: geminas totius orbis opulentissimas exasperauimus potestates, Constantinopolim et Persida: Oriens nos per terram territat, Occidens vero et terra et mari. Nam, ut alia omittam, Arthasium hactenus Antiochiae clypeus fuit: modo arcus intendit, modo in nos acuit sagittas. Nos pauci sumus, et semper tamen de paucis simus pauciores: valde imminutus est numerus noster, ubi unus amissus est comes Edessanus. Proinde uigilate, considerate attenti quid in tanto rerum cardine sit agendum: ego, quod sentio, breviter exponam. Exspectandae sunt nobis uires transmarinae: Galliarum populi concitandi: audacia illa aut nos liberabit, aut nulla». Pour une version française de cet extrait, voir Faits et gestes du Prince Tancrède..., éd. M. Guizot, 1825, p. 285 (nous avons un peu modifié la traduction). Une autre version du discours de Bohémond est dans The Ecclesiastical History..., éd. M. Chibnall, 19691980, Livre X, chap. 24, p. 378 : «Ab adolescentia mea irrequietus homo sum, et in laboribus vivens multa gravia perpessus sum, graviora quoque adhuc timeo me passurum. Nam michi certamen est cum imperatore, et cum paganis undique. Praterea votum vovi Domino cum essem in carcere, quod si liberarer ab ethnicorum ligamine, irem ad Sanctum Leonardum qui est in partibus Aquitaniae».

14. On trouvera plusieurs détails sur ce point dans Russo, 2002, p. 193-196.

15. Voir Russo, 2005, p. 3-42. 
plus grande prudence. Toutefois, il révèle la délicate situation de la principauté d'Antioche dans la première décennie du XII ${ }^{e}$ siècle: comme elle était, d'une part, coincée entre les belliqueux potentats islamiques des régions environnantes ${ }^{16}$, et, d'autre part, étranglée progressivement sur le plan économique et militaire par l'Empire byzantin ${ }^{17}$, le groupe dirigeant normand dut élaborer une politique d'endiguement des menaces extérieures, qui influença lourdement les choix politiques et institutionnels. En un mot, tous ces problèmes, ajoutés aux longues périodes de régence dues à l'absence d'une lignée princière solide, minée par des périodes répétées de minorité ${ }^{18}$, ont favorisé le retour d'Antioche dans la zone d'influence du royaume de Jérusalem, lequel dut intervenir à plusieurs reprises dans les régions de la Syrie du Nord. Cette situation de faiblesse politique était bien connue à la cour d'Antioche depuis l'époque de Roger de Salerne ${ }^{19}$, comme en témoigne l'incipit des Bella Antiochena élaboré par le chancelier Gautier, qui soulignait les prodiges réalisés par le prince-régent pour défendre les frontières de la principauté contre ses nombreux ennemis historiques ${ }^{20}$. Mais surtout Gautier, dans le deuxième livre, narre la sinistre bataille de l'Ager Sanguinis (1119), celle où les troupes chrétiennes furent massacrées par les troupes turkmènes conduites par Najm al-Dîn Il-Ghazi; Roger trouva la mort, et le narrateur lui-même fut capturé, si bien que fut mis en grave danger l'équilibre politico-institutionnel établi dans la région à

16. Voir The Damascus Chronicle..., éd. H.A.R. GibB, 2002, p. 54; Matthieu d’Édesse..., in Recueil des historiens des croisades, 1869, p. 73-74.

17. L'empire byzantin veut récupérer un territoire perdu dans les premières années du règne troublé d'Alexis I ${ }^{\text {er }}$ Comnène. Voir l'analyse brillante de Frankopan, 2013, p. 61 et suiv. Albert d'Aix..., éd. S.B. Edgington, 2007, Livre IX, chap. 47, p. 702, soutient que Bohémond était allé en France "ad adquirendas uires et commouendos principes aduersus Alexim regem Graecorum».

18. Il s'agit des périodes suivantes, 1100-1103: emprisonnement de Bohémond - régence de Tancrède; 1113-1126: minorité de Bohémond II, régence de Roger de Salerne (jusqu'en 1119), puis du roi Baudoin II de Jérusalem (jusqu’en 1126); 1130-1136: nouvelle régence et désaccords entre la princesse Alice et le roi de Jérusalem Foulques d'Anjou.

19. Selon Asbridge, 2000, p. 139-143, Roger prit le titre de prince sans aucune légitimité, et seule sa mort prématurée au combat l'empêcha de contester Bohémond II. Pour une réponse claire à cette question, voir Willelmi Tyrensis..., éd. R.B.C. Huygens, 1986, Livre XII, chap. 10, p. 558, 11. 21-29: «Dicitur autem hic idem princeps Rogerus homo fuisse perditissimus, incontinens, parcus et publicus adulter. Insuper etiam et dominum suum iuniorem Boamundum, senioris Boamundi filium, in Apulia cum matre morantem, ab Antiochia, que paterna ei hereditas erat, omni eo tempore, quo principatum tenuit, fecerat alienum: nam ea conditione dominus Tancredus, bone memorie, moriens ei principatum commiserat, ut reposcenti domino Boamundo vel eius heredibus non negaret» (voir Edbury et Rowe, 1988, p. 69); Fulcheri Carnotensis..., éd. H. HagenMeyer, 1913, Livre III, chap. III, 4, p. 623, nomme Bohémond II dominum de Roger; suivant Albert d'Aix..., éd. S.B. Edgington, 2007, Livre XII, chap. 9, p. 836, Roger fut nommé prince par le roi de Jérusalem Baudouin Ir ; dans Cafari Annales..., éd. L.T. Belgrano, 189o, p. 14, 11. 14-18, Roger n'est pas cité. Voir aussi The Damascus Chronicle..., éd. H.A.R. GiвB, 2002, p. 132; Kemal ed-Din..., in Recueil des historiens des croisades, 1884, p. 602.

20. Voir Galterii Cancellarii..., éd. H. Hagenmeyer, 1896, Livre I, Prologus, p. 61: «Operae pretium est audire et utilitati congruit, quomodo, quibus miraculis, qua gratia Deus arbiter bellum cum Parthis manu Rotgerii, principis Antiocheni, ex insperato gessit». On trouvera plusieurs éléments dans MaLleTt, 2010, p. 113-128, en particulier p. 114. Il est important de lire, sur cette question Walter the Chancellor's..., trad. T.S. AsBRIDGE, 1999, p. 1-17. 
la suite de la première croisade ${ }^{21}$. Pour compliquer encore la situation, on doit ajouter le fait que les princes normands furent confrontés à des ennemis de cultes différents; et même si les parties de chasse en commun étaient fréquentes entre chrétiens et musulmans - comme en témoignent les souvenirs d'un noble gentilhomme syrien de l'époque, originaire de Shaizar (Syrie nord-occidentale), le célèbre Oussama ibn Munqid ${ }^{22}$-, il régnait toujours un climat d'hostilité avec les Byzantins, et ce conflit devait exercer une forte influence sur l'Outremer. Par ailleurs, la mort au combat de Roger de Salerne (1113-1119) et celle de Bohémond II (1126-1130) ont souligné la fragilité des institutions politiques de la principauté d'Antioche: elle fut contrainte à plusieurs reprises de demander l'aide du royaume de Jérusalem, où les souverains disposaient d'une noblesse plus intéressée par les opérations militaires des régions voisines qu'aux équilibres politiques d'Antioche ${ }^{23}$. Dans les années trente du XII siècle eut lieu le passage de l'hégémonie normande à celle des Aquitains, favorisé par la mort tragique dans la bataille du jeune Bohémond II qui laissa la principauté sans héritier mâle ${ }^{24}$. Dans le cadre d'une politique dynastique menée par la cour de Jérusalem, la dynastie des Hauteville, régnant alors dans le Sud d'Italie avec Roger II, fut délibérément écartée de toute possibilité d'intervention. Un lignage de tradition croisée fut au contraire privilégié comme celui d'où provenait Raymond de Poitiers, fils du duc Guillaume IX d'Aquitaine, qui prit part à la croisade de 1101, et qui a épousé la jeune Constance en 1136 après un long bras de fer avec la veuve de Bohémond II, Alice de Jérusalem ${ }^{25}$.

\section{L'entourage normand}

Mais revenons au thème central de notre essai. Si on analyse le groupe de fidèles qui ont entouré Bohémond et Tancrède avant cette date - on doit à ce dernier

21. Sur ce point, on pourra aussi se reporter à Asbridge, 1997, p. 301-316 et Tyerman, 2012, p. 195-200; sur Il-Ghāzī, voir Hillenbrand, 1981, p. 250-292, en part. p. 276-278. Voir tout le deuxième livre des Galterii Cancellarii..., éd. H. Hagenmeyer, 1896, p. 78-115; voir aussi Fulcheri Carnotensis..., éd. H. Hagenmeyer, 1913, Livre III, chap. III, p. 620-624; Anonymi auctoris chronicon..., trad. A. AвоunA, 1974, chap. 280-281, p. 61-62; Damascus Chronicle..., éd. H.A.R. Gıвв, 2002, p. 159-161; Kemal ed-Din..., in Recueil des historiens des croisades, 1884, p. 616-619; Matthieu d'Édesse..., in Recueil des historiens des croisades, 1869, p. 122-124; Chronicle of Ibn al-Athir..., trad. D.S. RichARDS, 2005, p. 203-205.

22. Cf. Usāma ibn Munqid..., trad. M. CAssarino, 2001, p. 169. Voir Irwin, 1998, p. 71-87; Phillips, 2011, p. 38-46.

23. Sur les relations entre Antioche et Jérusalem, voir MAYER, 198o, p. 717-734.

24. Cf. Anonymi auctoris chronicon..., trad. A. AвounA, 1974, chap. 300, p. 77; Willelmi Tyrensis..., éd. R.B.C. HuYgens, 1986, Livre XIII, chap. 27, p. 623, ll. p. 1-12. Pour une description du jeune Bohémond, voir Matthieu d'Édesse..., in Recueil des historiens des croisades, 1869, p. 147 : «Il était de haute taille, à face de lion; il avait les cheveux de couleur blond clair».

25. Tyerman, 2012, p. 199-200; BARber, 2012, p. 152-153, 167-169; Hiestand, 1993, en part. p. 83-86; FrANCE, 2001, p. 87-101, en part. p. 96-97; en dernier lieu, Russo, 2014, p. 137-149. Voir aussi Riley-Smith, 1997, passim. Sur la croisade de Guillaume IX, voir Ligato, 2003, p. 31-103. Voir Anonymi auctoris chronicon..., trad. A. Aвоuna, 1974, chap. 302, p. 78 ; chap. 306, p. 80; chap. 309 , p. $81-82$; chap. 427 , p. 104 ; chap. 438 , p. 116. 
le plus grand engagement dans la construction politique de la principauté naissante d'Antioche, il apparaît clairement qu'une grande partie de ses membres se targuait d'avoir une ascendance "normande" au sens large: sur un total de 25, nous avons en fait 9 Normands de Normandie ou d'Italie du Sud, 5 non-Normands (3 sont les épouses de princes qui se sont succédé durant ces années), et 11 cas sur lesquels on ne peut se prononcer faute de plus amples détails ${ }^{26}$. Il est frappant de constater, cependant, que les deux ecclésiastiques appartenant au groupe de commandement, Bernard de Valence et Pierre de Narbonne, provenaient d'autres régions transalpines, comme si le manque d'ecclésiastiques dans le contingent normand ${ }^{27}$ avait favorisé le recrutement dans d'autres zones géographiques, un fait qui trouve des similitudes en Sicile à l'époque de la conquête normande ${ }^{28}$. Cependant, en élargissant la vision analytique, on en vient plutôt à penser que les ecclésiastiques pouvaient se déplacer plus facilement d'un contingent de croisés à l'autre et qu'il leur était plus facile de suivre un seigneur différent plutôt que d'autres pèlerins ${ }^{29}$. Par exemple Arnulf, évêque de Martirano (province de Cosenza), partit sûrement avec les Normands dans le Sud, mais s'associa ensuite au contingent lorrain pour continuer le voyage vers la ville sainte, interrompu par Bohémond et ses compagnons; après sa capture par quelques musulmans au lendemain de la conquête de Jérusalem, nous n'avons plus aucune trace de lui, malgré les différentes hypothèses formulées au sujet de son destin par les chroniqueurs de l'époque $^{30}$. D'autres cas confirment l'impression d'une telle mobilité. Le plus célèbre est certainement celui représenté par le chroniqueur Foucher de Chartres : parti à la suite du comte Étienne de Blois, puis rejoignant le contingent lorrain en tant que chapelain de Baudouin de Bouillon, celui-ci parvint à recouvrir les mêmes fonctions à la cour de Jérusalem, devenant le "chantre» par excellence des succès du royaume de Jérusalem ${ }^{31}$. Encore plus sensationnelle est l'ascension de Bernard de Valence; parti à Jérusalem comme chapelain à la suite de l'évêque Adhémar du Puy ${ }^{32}$, il fut ensuite consacré

26. Ici, nous tirons les informations de AsBRIDGE, 2000, p. 163-168: «it is likely that a high proportion of these [scil. les hommes du prince d'Antioche] came from a Norman background» (p. 168)

27. Cf. Hystoria de via..., éd. E. D’Angelo, 2009, chap. V, 29, p. 20, qui mentionne un «episcopus de Ariano" parmi les Normands d'Italie du Sud, qu'on trouve aussi dans Willelmi Tyrensis..., éd. R.B.C. Huygens, 1986, Livre IX, chap. 14, p. 439, 11. 31-32.

28. Cf. De' Giovanni-Centelles, 2007, p. 207-234.

29. Nous sommes tout à fait d'accord avec Murray, 1997, p. 352, quand il dit: "it is clear that none of the armies on the crusade were static entities, but changed constantly».

30. On trouvera plusieurs détails sur ce point dans Gesta Francorum..., éd. R. HiLl, 1962, chap. XXXIX, p. 93-94; The Historia Ierosolimitana..., éd. S. BIdDlecombe, 2014, Livre IV, p. 115 ( dubium est an abductus an peremptus»); une vision plus critique est donnée par Guillaume de Tyr: Willelmi Tyrensis..., éd. R.B.C. Huygens, 1986, Livre IX, chap. 12, p. 340, 11. p. 50-56. La tradition épique postérieure mit en exergue l'évêque de Martirano : cf. The Old French Crusade..., éd. N.R. Thorpe, 1992, sous le nom de Mautran, Maltran. Sur Arnulf, voir Loud, 1992, p. 49-50; mais surtout Rowe, 1957, p. 472-473.

31. Sur Foucher, voir Russo, 2010, p. 115-117.

32. Sur les clercs qui suivent l'évêque du Puy, voir Russo, 2006, p. 820. Sur Bernard de Valence: Radulphus Cadomensis..., éd. E. D’Angelo, 2011, chap. 408, p. 118, 11. 4012-4015; Ibid., chap. 427 , 
évêque du siège ecclésiastique d'Artah et quelques mois plus tard, durant l'été 1100, il fut intronisé patriarche à Antioche grâce à l'appui de Bohémond: il profita du retrait - très probablement contraint - du patriarche orthodoxe Jean V l'Oxite à Constantinople, qui marqua le passage de l'hégémonie de l'Église orthodoxe à celle de l'Église catholique, autre élément de friction avec la cour de Byzance ${ }^{33}$.

Mettant de côté l'analyse quantitative, nous notons, à la lecture des tout premiers documents des princes d'Antioche - il s'agit de deux privilèges émis en faveur des Génois - qu'apparait clairement l'extrême mobilité de l'entourage princier naissant ${ }^{34}$. Robert de Sourdeval, Robert d'Anzi, Raoul le Roux et Boel de Chartres sont aux côtés de Bohémond en $1098^{35}$. Nous possédons plus d'informations sur les deux premiers: Robert de Sourdeval aurait poursuivi sa carrière dans la principauté d'Antioche avant d'être tué dans la bataille de Tell Danith (1115), laissant des héritiers documentés à Antioche pendant deux générations ${ }^{36}$; quant à Robert d'Anzi, il aurait rejoint le contingent lorrain conduit par Godefroy de Bouillon, puis il reçut des rentes près d'Arsuf et finit par s'intégrer de façon stable à la noblesse du royaume de Jérusalem ${ }^{37}$. Cependant, seulement trois ans plus tard, en 1101, l'entourage princier à Antioche semble avoir radicalement changé: aux côtés du régent Tancrède, nous trouvons en fait Guillaume Carpenter, Richard Le Connétable ${ }^{38}$, Guillaume Acatapano, Roger de Florence et Guillaume Malconvento ${ }^{39}$. Une autre histoire est racontée

p. 123, 1l. 4224-4226; Willelmi Tyrensis..., éd. R.B.C. Huygens, 1986, Livre VI, chap. 23, p. 340, 11. 38-42; Livre VII, chap. 8, p. 353, 1l. 15-19; Livre XIV, chap. 10, p. 641, 11. 1-4. Pour plus de précisions, voir НAMILTON, 1980, p. $21 \mathrm{sq}$.

33. Cf. Rey, 1900-1901, p. 131-133; mise à jour dans Fedalto, 1970, p. 116-119; Asbridge, 2000, p. 198-213; Hamilton, 2006, p. 173. Dans un document en faveur des Hospitaliers, Bernard se nommerait «Dei gratia Antiochene ecclesie primus patriarcha Latinus»: cf. Cartulaire général..., éd. J. Delaville Le RoulX, 1894, p. 9 (doc. 5). Sur le patriarche orthodoxe, voir Gautier, 1964, p. 128-157; enfin MACEvitT, 2008, p. 110-112. Une hypothèse fort intéressante est avancée par Shepard, 2012, p. 363-366, qui soutient que Jean était un agent byzantin dans Antioche.

34. Sur la question, voir MARTIN, 2002, p. 231-232.

35. À l'exception de Raoul le Roux, tous les autres sont attestés aux côtés de l'aîné de Robert Guiscard jusqu'aux origines de l'expédition vers Jérusalem: voir Gesta Francorum..., éd. R. HiLL, 1962, chap. IV, p. 7-8: «Tandem transfretavit mare cum suo exercitu [scil. de Bohémond], et cum eo Tancredus Marchisi filius, et Richardus princeps, ac Rainulfus frater eius, et Rotbertus de Ansa, et Hermannus de Canni, et Rotbertus de Surda Valle, et Robertus filius Tostani, et Hunfredus filius Radulfi, et Ricardus filius comitis Rainulfi, et comes de Russignolo cum fratribus suis, et Boello Carnotensis, et Alberedus de Cagnano, et Hunfredus de Monte Scabioso" [souligné par l'auteur]. Les trois sont encore mentionnés dans l'Hystoria de via..., éd. E. D’ANGelo, 2009, chap. V, 28, p. 19-20. Pour plus de précisons, voir Jamison, 1939, p. 183-208, 195 sq.

36. Cf. Galterii Cancellarii..., éd. H. Hagenmeyer, 1896, Livre I, chap. VI, p. 75; Martin, 2002, p. 236; AsBRIDGE, 2000, p. 165-166.

37. Voir Murray, 1997, p. 353, 356.

38. Sur le connétable dans la principauté d'Antioche voir AsBridge, 200o, p. 184.

39. Cf. Liber privilegiorum..., éd. D. Puncuh, 1962, doc. 23 et 25, p. 40-42. Déjà édités dans Italia Sacra..., éd. F. Ughelli, 1719, vol. IV, coll. 846-848. Voir aussi Cafari Annales..., éd. L.T. Belgrano, 189o, p. 113-114. Favreau-Lilie, 1988, p. 327-381, a montré que le document de Tancrède de 1101 est interpolé, mais l'identité des souscripteurs est - à mon avis - indéniable. En dernier lieu, il faut voir MUSARRA, à paraître, chap. IV, 2. 
en 1108 dans deux actes rédigés en faveur des Pisans pour expliquer l'aide navale apportée lors du siège contre le port byzantin de Laodicée ${ }^{40}$. Dans ces actes, apparaissent en tant que témoins - en plus du patriarche Bernard et d'un petit groupe d'évêques et abbés - trois aristocrates ayant reçu le titre de barons: cela vient confirmer la présence d'un groupe aristocrate plus structuré autour de Tancrède, vigoureux continuateur de la politique d'indépendance menée par Bohémond ${ }^{41}$.

Il est cependant extrêmement difficile, à la lumière de la documentation en notre possession, de faire des déductions qui vont au-delà d'un certain degré de probabilité étant donné que les documents qui nous sont parvenus proviennent d'entités externes à la principauté d'Antioche, qu'ils soient l'expression d'intérêts commerciaux de la communauté de marchands d'origine italienne ou d'actes patrimoniaux établis à l'avantage d'institutions ecclésiastiques de Jérusalem ${ }^{42}$. C'est l'une des raisons de la prédominance des recherches sur le royaume de Jérusalem, qui a souvent été le modèle de référence pour les chercheurs des structures politiques et administratives de l'Orient latin, et a empêché l'identification des caractéristiques d'un modèle "normanno-antiochien». Une telle situation explique aussi le besoin ressenti par les chercheurs de combler les lacunes en insérant des éléments documentaires issus du royaume de Jérusalem dans la grille politico-institutionnelle d'Antioche, afin de rendre compte de son évolution interne. Il suffit de passer au peigne fin la longue liste des regestes sur le royaume de Jérusalem édités à la fin du XIX ${ }^{\mathrm{e}}$ siècle par Reinhold Röhricht pour s'en rendre compte: tous les faits de l'histoire de la principauté d'Antioche sont mentionnés dans l'œuvre fondamentale de l'érudit allemand et attestent le manque de documents à notre disposition aujourd'hui. En même temps, cela confirme l'attrait du modèle de Jérusalem qui a concentré l'attention de tous les spécialistes ${ }^{43}$, une situation déjà remarquée il y a plus d'un siècle par Emmanuel Guillaume Rey dans ses recherches pionnières sur la principauté d'Antioche: «Les historiens des croisades, toujours préoccupés de Jérusalem ont, pour ainsi dire, systématiquement laissé dans l'ombre l'histoire de la principauté d'Antioche ${ }^{44}$.

Cependant la particularité politique d'Antioche saute aux yeux de l'historien comme en témoigne un sceau exceptionnel de Thierry de Barneville,

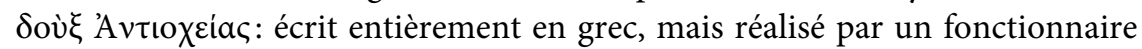
occidental des nouvelles autorités normandes, il illustre clairement l'expérience

40. Voir Cafari Annales..., éd. L.T. Belgrano, 189o, p. 114, 11. 13-20.

41. Cf. Carte dell'archivio..., éd. M.T. CARLI, 1969, doc. 37-38, p. 80-83: «et testimonio baronum horum scilicet R(oberti) Fulconis filii et Engellerii et R(oberti) sancti Laudi» (p. 81, 11. 13-15); "et testimonio baronum domni Tancredi horum R(oberti) Fulconis filii et Engellerii et $R$ (oberti) sancti Laudi» (p. 83, 11. 16-17). Le dernier est aussi présent dans un document écrit en 1118 à côté du prince-régent Roger de Salerne: cf. Cartulaire général..., éd. J. Delaville Le RoulX, 1894, p. 38 (doc. 45). Voir Favreau-Lilie, 1988, p. 111-116.

42. On trouvera une discussion très intéressante dans Asbridge, 1999, p. 313-316. Voir aussi les recherches de REY, 1900-1901, passim.

43. Cf. Regesta Regni Hierosolymitani..., éd. R. RöHricht, 1893, doc. 2, 5-7, 9-14, 16, 33, 35, 53, 66, 70, 72-73, 76, 86, 119 (pour les actes avant 1130).

44. ReY, 1896, p. 321. 
acquise par les Normands dans le sud de l'Italie au contact de l'administration byzantine, qui a fait du sceau de plomb l'élément de validation des documents par excellence ${ }^{45}$. Par ailleurs, ce n'est pas le fait du hasard si le même personnage est mentionné dans une source historique sous le nom de Típı $\delta \varepsilon \Pi \alpha \rho v \alpha \beta \iota \lambda \lambda(\varepsilon)^{46}$, une hellénisation de Thierry effectuée incorrectement, mais qui évidemment trahit le fort substrat grec de l'auteur du texte. Il s'agit d'une autre confirmation de l'environnement cosmopolite de la principauté d'Antioche ${ }^{47}$, avec lequel les dirigeants politiques normands durent traiter immédiatement, alors qu'ils étaient hégémoniques du point de vue politique et militaire malgré leur infériorité numérique; et l'on en trouve encore des confirmations dans les traductions de l'arabe en latin faites justement pendant les années ici décrites et bien étudiées par Charles Burnett. La principauté d'Antioche était une réalité urbaine particulièrement animée du point de vue culturel: on peut aujourd'hui en comprendre la mesure grâce à une série d'études détaillées, menées au cours des dernières décennies sur l'activité de scientifiques de l'importance d'Adalard de Bath et de traducteurs comme Étienne de Pise. Antioche représente un véritable avant-poste oriental de l'Occident médiéval au cours de la première moitié du $\mathrm{XII}^{\mathrm{e}}$ siècle, pendant laquelle les échanges culturels enrichirent substantiellement le patrimoine culturel du continent européen ${ }^{48}$.

\section{Conclusion}

La rencontre entre les chevaliers occidentaux arrivés à la suite de la soi-disant «première croisade» et les populations locales - qu'elles soient chrétiennes ou musulmanes - a été interprétée de façon très variable par l'historiographie de l'époque moderne, qui s'est souvent appuyée sur un concept d'identité religieuse qui s'applique mal aux sources analysées ici ${ }^{49}$. Les Normands qui ont dominé la scène politique de la principauté d'Antioche au cours des trois premières décennies du $\mathrm{XII}^{\mathrm{e}}$ siècle ont été confrontés à une population majoritaire avec laquelle ils ont dû établir des relations de collaboration, s'ils voulaient garantir un avenir à leur projet politique. Cependant, la documentation à notre disposition - en grande partie basée sur des chroniques écrites par des personnes proches

45. Cheynet, 1984, p. 223-228. Pour Oiкonomides, 1983, p. 147-157: «the lead seal was considered an important element of an act because it guaranteed its validity, even if it was not accompanied by the subscription of the author» (p. 147). Par ailleurs, LAPINA, 2015, a souligné à juste titre l'influence byzantine sur les monnaies d'Antioche au XII ${ }^{\mathrm{e}}$ siècle.

46. Cf. Galterii Cancellarii..., éd. H. HAGENMEyer, 1896, Livre I, chap. V, p. 72 (voir aussi la note 22, p. 182). Cf. Asbridge, 200o, p. 166.

47. Cf. Galterii Cancellarii..., éd. H. Hagenmeyer, 1896, Livre I, chap. I, p. 63, qui rappelle que les habitants d'Antioche dans l'année 1115 étaient «Latini, Graeci, Syri, Armeni, aduenae et peregrini». Voir aussi MAcEvitT, 2008, p. 63-65.

48. Des précisions dans Burnett, 2000, p. 1-78, en part. 1-19. Il soutient que "the Antioch-Pisa connection was a conduit through which Arabic works arrived in Europe» (p. 15). Voir aussi Edgington, 2006, p. 247-259. On trouvera un autre avis dans Tyerman, 2012, p. 241-242.

49. MACEvITT, 2008, p. 179 (en particulier: «While boundaries between different religious communities did exist, they were permeable and elastic markers that allowed for a variety of religious encounters"). 
des centres du pouvoir (la cour d'Antioche dans le cas de Raoul de Caen et Gautier le Chancelier, celle de Jérusalem pour Foucher de Chartres et Guillaume de Tyr) - donne l'image d'un monde belliqueux, radicalement opposé à la fois à un monde islamique fragmenté et à l'Empire byzantin, et cette hostilité constituera l'héritage politique le plus lourd laissé par Bohémond de Hauteville à ses successeurs. Au contraire, il reste peu de traces du pragmatisme dont ont fait preuve les Normands au cours du XII ${ }^{e}$ siècle, et c'est seulement aujourd'hui que les historiens commencent à le mettre en évidence ${ }^{50}$.

Si l'on considère le thème historiographique de la diaspora normande des $\mathrm{XI}^{\mathrm{e}}$ et XII ${ }^{\mathrm{e}}$ siècles, le cas d'Antioche délivre plusieurs enseignements. D'une part, il faut toujours être très prudent en ce qui concerne la documentation sur laquelle on s'appuie pour reconstituer les événements de l'expansion normande: partant de l'Angleterre, en continuant vers le sud de l'Italie, et arrivant dans l'Orient latin, on assiste à une progressive désagrégation des preuves documentaires en notre possession à cause de l'anéantissement de la principauté d'Antioche par les Mamelouks en $1268^{51}$. L'historien de l'Angleterre normande peut en effet s'appuyer sur une série de documents d'une importance et d'une richesse exceptionnelles (il suffit de penser seulement au Domesday Book ou bien aux Pipe Rolls qui débutent à partir de 1129-1130), qu'on ne possède pas pour d'autres régions dans lesquelles eurent lieu les exploits des chevaliers normands. Sous l'étiquette $d^{\prime}$ '«expansion normande» se cache une très forte disparité des événements et des contextes qui témoigne de la grande capacité d'adaptation des chevaliers du Nord, mais aussi de la difficulté d'uniformiser l'expérience politico-militaire comme l'historiographie a voulu l'expliquer dans le passé ${ }^{52}$.

D’autre part, si l'on repense aux événements de la principauté d'Antioche, nous notons que son siège patriarcal a été occupé au cours des trente premières années par un clerc d'une région occitane, qui était destiné à une carrière médiocre s'il était resté en Occident (Bernard de Valence) ${ }^{53}$, et que divers princes et régents d'Antioche épousèrent des dames nobles n'appartenant pas au monde normand. Il faut ajouter qu'une partie des pèlerins embarqués à la suite de Bohémond (l'évêque Arnoul de Martirano, Robert d'Anzi) se lia à d'autres seigneurs, que même des aristocrates originaires du Massif Central (les Mazoir) partis à la suite de Raymond IV de Saint-Gilles ${ }^{54}$, implacable ennemi de Bohémond, apparaissent ensuite dans la documentation d'Antioche comme des fidèles de la maison princière, grâce aussi à la conservation de leurs

50. On pourra consulter Hillenbrand, 1981, p. 284 sq. Mais il faut surtout lire Houben, 2013, p. 109-116.

51. Tyerman, 2012, p. 736, 821-826.

52. Par exemple Brown, 2010, p. 186, envisage, dans la fondation de la principauté d'Antioche, « un'altra dimostrazione della normanitas» [souligné par l'auteur]; plus pertinent: CHibNALL, 2005, p. 141 et suiv.

53. Hamilton, 1980, p. 22, soutient que «A similar man [scil. Bernarde de Valence] in western Europe, unless he had the backing of very powerful patrons, would probably have had an undistinguished career» [souligné par l'auteur].

54. Voir Hill et Hill, 1959, passim. 
archives dans celles de l'ordre de l'Hôpital ${ }^{55}$. Tout cela ne fait que démontrer l'hybridation présente dans le groupe dirigeant dès les premières étapes de l'affirmation de la nouvelle principauté. Il y a quelques années, Alan V. Murray s'est demandé dans quelle mesure la principauté d'Antioche était normande: il a conclu que le concept de Normanitas pourrait s'appliquer aussi à l'Orient latin, malgré ce que laissent transparaittre les chroniqueurs de l'époque ${ }^{56}$. Il serait opportun de reconsidérer les caractéristiques politiques et institutionnelles d'un grand centre urbain comme Antioche, et d'insister fortement sur le contexte politique environnant: immergé dans une réalité de "guerre permanente», le petit groupe dirigeant fidèle à la dynastie normande dut nécessairement tisser des liens d'alliance avec tous les groupes ethniques locaux - comme les Arméniens $s^{57}$ - désireux d'établir des formes de coopération avec les nouveaux arrivants. Tout ceci explique le témoignage du patriarche jacobite local, Michel le Syrien, selon lequel les croisés à Antioche maintenaient des relations pacifiques avec toutes les confessions chrétiennes, octroyant églises et monastères avec une extrême libéralité, indépendamment des questions théologiques et doctrinales qui divisaient les différentes communautés chrétiennes ${ }^{58}$. Dans ce contexte, la mise en avant d'une normannité devait se révéler peu utile pour la construction d'une unification politique, car le grand nombre de fidèles gravitant autour des nouveaux seigneurs d'Antioche pouvait difficilement se réclamer d'une identité normande ${ }^{59}$.

Dans la célèbre Tabula Peutingeriana datant de l'Antiquité tardive, puis retouchée au Moyen Âge, Antioche est représentée comme une femme auréolée, assise sur un trône et tenant une lance dans la main droite, avec à ses côtés un jeune garçon représentant le fleuve Oronte, tandis qu'un temple et un aqueduc complètent l'illustration graphique de la ville ${ }^{60}$. Seule avec Rome et Constantinople à jouir d'une telle personnification, un copiste anonyme postérieur accentua les dimensions de la représentation de la ville d'Antioche au point de la surdimensionner par rapport à Rome ${ }^{61}$. C'est dans cette grande et

55. Martin, 2002, p. 233; plusieurs détails dans CAHEN, 1940, p. 535-538; Asbridge, 2000, p. 161-162. Cafari Annales..., éd. L.T. Belgrano, 189o, p. 115, 11. 16-18, cite pour l'année 1101 "Rainaldus Mansuer nomine, alterius Raynaldi filius, constabularii Antioceni principis, et dominus erat Vananae et Marachiae» (voir la note 8 au texte, mais l'identification est refusée dans CAHEN, 1940, note 16, p. 279-280).

56. Murray, 1997, p. 359. Sur le concept historiographique de Normanitas voir Loud, 1982; ID., 2002, et en dernier lieu CHLÉIRIGH, 2013.

57. Sur les relations entre les Arméniens et les Croisés, voir Forse, 1991, p. 13-22; Holt, 2004, p. 23-26; DÉDÉYAN, 1996, p. 79-88; Mutafian, 1996, p. 453-461.

58. Cf. Chronique de Michel le Syrien..., éd. J.-B. Снавот, 1905, Livre XV, chap. VII, p. 183. Sur son patriarcat (1166-1199) voir REY, 1900-1901, p. 152-153. En général, sur cette question, voir Papadakis, Meyendorff, 1994, p. 107 et suiv.

59. L'utilité conceptuelle du terme Normanitas a été fort critiquée par David Bates (BATES, 2013, p. $1-7$, p. $185-190)$.

60. La meilleure reproduction est dans le magnifique facsimile Tabula Peutingeriana, 1976, segment IX; voir aussi Tabula Peutingeriana, 2003, p. 11, 46. En général, voir SANSONE, 2009, p. 78-83.

61. TAlBERT, 2010, p. 124: "Post-original too, in my view, is the creation of an exceptionally visible symbol for Antiochia, rendered larger than that for Rome itself in fact, the map's intended focal point». 
riche ville, pleine de traditions romaines et chrétiennes séculaires bien rappelées par Guillaume de $\mathrm{Tyr}^{62}$, que les Normands arrivèrent et régnèrent pendant plus de trente ans. Le mouvement d'expansion normande, cependant, était arrivé à ses limites extrêmes: de là sa durée éphémère.

\section{Bibliographie}

\section{Sources}

Albert d'Aix, Historia Ierosolimitana, éd. S.B. Edgington, Oxford, Clarendon Press, 2007.

Anonymi auctoris chronicon ad A.C. 1234 pertinens, t. II, trad. A. AвounA, (CSCO t. 354, Scriptores Syri t. 154), Louvain, Secrétariat du CorpusSCO, 1974 [= Anonymi auctoris chronicon ad annum Christi 1234 pertinens, t. II, éd. I.-B. СНАВот, (CSCO t. 82, Scriptores Syri t. 37), Louvain, Imprimerie Orientaliste L. Durbecq, 1916].

Cafari Annales, in Annali genovesi di Caffaro e de' suoi continuatori, éd. L.T. Belgrano, (Fonti per la Storia d'Italia, 11), Gênes, ISIME, 1890.

Carte dell'archivio capitolare di Pisa (1101-1120), vol. 4, éd. M.T. CARLI, (Thesaurus Ecclesiarum Italiae, VII/ 4), Rome, Edizioni di storia e letteratura, 1969.

Le Cartulaire du chapitre du Saint-Sépulcre de Jérusalem, éd. G. BresC-Bautier, (Documents relatifs à l'histoire des croisades, XV), Paris, Paul Geuthner, 1984.

Cartulaire général de l'ordre des Hospitaliers de Saint-Jean de Jérusalem (110o-1310), éd. J. Delaville Le RoulX, vol. I, Paris, Leroux, 1894.

Chartes de l'abbaye de Notre-Dame de la vallée de Josaphat en Terre Sainte (1108-1291), éd. C. KoHLER, in Archives de l'Orient Latin, VII, 1899, p. 108-222.

The Chronicle of Ibn al-Athir for the Crusading Period from al-Kamil fi'l-Ta'rikh, vol. I, trad. D.S. Richards, Aldershot, Ashgate, 2005.

Chronique de Michel le Syrien patriarche jacobite d'Antioche (1166-1199), éd. J.-В. Снавот, t. III, Paris, Leroux, 1905.

Codice Diplomatico della Repubblica di Genova, éd. C. Imperiale di SANT'Angelo, vol. I, (Fonti per la Storia d'Italia, 77), Rome, ISIME, 1936.

The Damascus Chronicle of the Crusades. Extracted and translated from the Chronicle of Ibn Al-Qalānisī, éd. H.A.R. GıвB, rist. New York, Dover, 2002 [éd. orig. Londres, Luzac \& Co., 1932].

Domesday Book. A Complete Translation, éd. A. Williams - G.H. Martin, Londres, Penguin, 2002.

The Ecclesiastical History of Orderic Vitalis, éd. M. Chibnall, (Oxford Medieval Texts), Oxford, Clarendon Press, 1969-1980.

62. Willelmi Tyrensis..., éd. R.B.C. Huygens, 1986, Livre IV, chap. 9, p. 244-245, décrit ainsi la ville d'Antioche: «civitas gloriosa et nobilis, tercium vel potius secundum - nam de hoc maxima questio est - post urbem Romam dignitatis gradum sortita, omnium provinciarum, quas tractus continet Orientalis, princeps et moderatrix» (ibid., p. 244, 11. 1-4). L'intérêt commercial d'Antioche est souligné dans VORDERSTRASSE, 2010, p. 151-171. 
Faits et gestes du Prince Tancrède pendant l'expédition de Jérusalem par Raoul de Caen, éd. M. Guizot (Collection des mémoires relatifs à l'histoire de France), Paris, 1825 .

Fulcheri Carnotensis Historia Hierosolymitana (1095-1127), éd. H. HagenMeyer, Heidelberg, C. Winter, 1913.

Galterii Cancellarii Bella Antiochena, éd. H. HagenMeyer, Innsbruck, 1896.

Gesta Francorum et aliorum Hierosolimitanorum, éd. R. Hill, (Medieval Texts), Londres, Clarendon Press, 1962.

The great roll of the pipe for the thirty first year of the reign of King Henry I, Michaelmas 1130, éd. J.A. Green, (Pipe Roll Society, new series 57), Londres, The Pipe Roll Society, 2012.

The Historia Ierosolimitana of Baldric of Bourgueil, éd. S. BidDlecombe, Woodbridge, Boydell, 2014.

Hystoria de via et recuperatione Antiochiae atque Ierusolymarum (olim Tudebodus imitatus et continuatus), éd. E. D’Angelo, (Edizione Nazionale dei testi Mediolatini, 23), Florence, SISMEL, 2009.

Italia Sacra sive de episcopis Italiae et insularum adjacentium, éd. F. UGHELLI, vol. IV, Venetiis, 1719.

Kemal ed-Din, Extraits de la Chronique d'Alep, in Recueil des historiens des croisades, Historiens orientaux, III, Académie des inscriptions et Belles-Lettres, Paris, Imprimerie nationale, 1884 , p. 571-69o.

Liber privilegiorum ecclesiae ianuensis, éd. D. PuncuH, Gênes, In palatio archiepiscopali ianuensi, 1962.

Matthieu d'Édesse, Chronique. Extraits, in Recueil des historiens des croisades, Documents arméniens, I, Académie des Inscriptions et Belles-Lettres, Paris, Imprimerie nationale, 1869, p. 1-150.

The Old French Crusade Cycle. VI. La Chanson de Jérusalem, éd. N.R. Thorpe, Tuscaloosa - Londres, University of Alabama Press, 1992.

Radulphus Cadomensis Tancredus, éd. E. D’Angelo, Corpus Christianorum, Cont. Mediaevalis, t. CCXIII, Turnhout, Brepols, 2011.

Regesta Regni Hierosolymitani (1097-1291), éd. R. RöHRICHT, Berlin, Libraria Academica Wagneriana, 1893.

Richard Fitz Nigel, Dialogus de scaccario, éd. C. Johnson, Oxford, Oxford University Press, 1983.

Tabula Peutingeriana. Codex Vindobonensis 324 (Vollständige Faksimile - Ausgabe im original format), Graz, Akademische Druck, 1976.

Tabula Peutingeriana. Le Antiche vie des mondo, Florence, Olschki, 2003.

Usāma ibn Munqid, Le lezioni della vita. Un cavaliere siriano e le Crociate, M. CAssARINO (trad. it.), Milan, Ariele, 2001.

Walter the Chancellor's The Antiochene Wars, T.S. Asbridge (trad.), Aldershot, Ashgate, 1999, p. 1-17.

Willelmi Tyrensis archiepiscopi Chronicon, éd. R.B.C. Huygens, Corpus Christianorum, Cont. Mediaevalis, t. LXIII-LXIII A, Turnhout, Brepols, 1986. 


\section{Études}

Amouroux-Mourad, Monique, Le comté d'Edesse: 1098-1150, Paris, Paul Geuthner, 1988.

Asbridge, Thomas S., "Alice of Antioch: a case study of female power in the twelfth century», in The Experience of Crusading: Defining the Crusader Kingdom, vol. 2, éd. P. Edbury, J. Phillips, Cambridge, Cambridge University Press, 2003, p. 29-47.

AsBridge, Thomas S., The Creation of the Principality of Antioch, 1098-1130, Woodbridge, Boydell, 2000

Asbridge, Thomas S., "The "Crusader" Community at Antioch: the impact of Interaction with Byzantium and Islam», in Transactions of the Royal Historical Society, 6 a., 9 (1999), p. 305-325.

AsBridge, Thomas S., «The significance and causes of the battle of the Field of Blood», Journal of Medieval History, 23 / 4 (1997), p. 301-316.

BArber, Malcolm, The Crusader States, Londres, Yale University Press, 2012.

Bates, David, The Normans and Empire, Oxford, Oxford University Press, 2013.

Bates, David et BAuduin, Pierre (éd.), 911-2011 : Penser les mondes normands médiévaux, Caen, Presses universitaires de Caen, 2016.

Brown, Allen R., Storia dei Normanni, Bologne, Odoya, 2010 ( $2^{\mathrm{e}}$ éd.; orig. Woodbridge, 1984).

BurnetT, Charles, «Antioch as a link between Arabic and Latin culture in the twelfth and thirteenth centuries", in Occident et Proche-Orient: contacts scientifiques au temps des croisades, éd. I. Draelants, A. Tihon et B. Van den Abeele, Turnhout, Brepols, 2000, p. 1-78.

Cahen, Claude, La Syrie du Nord à l'époque des croisades et la principauté franque d'Antioche, Paris, Paul Geuthner, 1940.

CAhen, Claude, «Un document concernant les Melkites et les Latins d'Antioche au temps des Croisades", Revue des études byzantines, 29 (1971), p. 285-292.

Carpenter, Donald, The Struggle for Mastery. Britain 1066-1284, Londres, Penguin (The Penguin History of Britain, vol. 2), 2003.

Cheynet, Jean-Claude, "Le sceau de Thierry de Barneville», duc d'Antioche, Revue numismatique, $6^{\mathrm{e}}$ série, 26 (1984), p. 223-228.

Chibnall, Marjorie, Anglo-Norman England, 1066-1166, Oxford, Blackwell, 1993 ( $2^{\mathrm{e}}$ éd.).

Chibnall, Marjorie, I Normanni. Da guerrieri a dominatori, Gênes, ECIG, 2005 (éd. orig. Oxford 2000).

Chléirigh, Léan Ni, «Gesta Normannorum? Normans in the Latin chronicles of the First Crusade», in Norman Expansion. Connections, Continuities and Contrasts, éd. K.J. Stringer et A. Jotischky, Farnham, Ashgate, 2013, p. 207-226.

Clanchy, Michael T., England and its Rulers: 1066-1307, Oxford, Blackwell, 2006 ( $3^{\mathrm{e}}$ éd.).

Clanchy, Michael T., From Memory to Written Record. England 1066-1307, Oxford Cambridge, Blackwell, 2012 ( $3^{\text {e éd.). }}$ 
DÉDÉYAN, Gérard, «Les princes arméniens de l'Euphratèse et l'Empire byzantin (fin XI'-milieu XII ${ }^{\mathrm{e}} \mathrm{s}$.)", in L'Arménie et Byzance. Histoire et culture (Byzantina Sorbonensia 12), Paris, Publications de la Sorbonne, 1996, p. 79-88.

DE’ Giovanni-Centelles, Guglielmo, «Croce e spada nella Sicilia del Gran Conte: le nomine vescovili. Processi di ricristianizzazione mediterranea nell'XI secolo», in Ruggero I Gran Conte di Sicilia, 1101-2001, éd. G. DE' Giovanni-Centelles, Rome, Istituto Italiano dei Castelli - Sezione Sicilia, 2007, p. 141-274.

Edbury, Peter W., The Assises d'Antioche: law and custom in the principality of Antioch, in Norman Expansion. Connections, Continuities and Contrasts, éd. K.J. STRINGER et A. Jотіsснку, Farnham, Ashgate, 2013, p. 241-248.

Edbury, Peter W. et Rowe, John G., William of Tyre. Historian of the Latin East, Cambridge, Cambridge University Press, 1988.

Edgington, Susan B., "Antioch: Medieval City of Culture", in East and West in the Medieval Eastern Mediterranean, vol. 1, éd. K. Ciggaar et M. Metcalf, Peeters, Leuven, (Orientalia Lovaniensa Analecta, 147), 2006, p. 247-259.

Favreau-Lilie, Marie-Luise, Die Italiener im Heiligen Land: vom ersten Kreuzzug bis zum Tode Heinrichs von Champagne (1098-1197), Amsterdam, Hakkert, 1988.

FedAlto, Giorgio, «L'origine della gerarchia ecclesiastica latina nei patriarcati di Antiochia e di Gerusalemme, durante la Prima Crociata (1097-1099)», Studia Patavina, XVII (1970), p. 100-120.

Figlivolo, Bruno, «Chiesa e feudalità nei principati latini d'Oriente durante il XII secolo", in Chiesa e mondo feudale nei secoli X-XII. Atti della dodicesima settimana internazionale di studio della Mendola, Milan, Vita e Pensiero, 1995, p. 375-409.

Flori, Jean, Bohémond d'Antioche. Chevalier d'aventure, Paris, Payot, 2007.

Forse, James H., «Armenians and the First Crusade», in Journal of Medieval History, 17 (1991), p. 13-22.

FranCE, John, «The Normans and Crusading», in The Normans and their Adversaries at War, éd. R.P. ABELs et B.S. BACHRACH, Woodbridge, Boydell, 2001, p. 87-101.

Frankopan, Peter, La prima crociata. L'appello da Oriente, Milan - Turin, Bruno Mondadori, 2013 [éd. orig. Londres 2012].

Gautier, Paul, «Jean V l'Oxite, patriarche d'Antioche. Notice biographique», in Revue des études byzantines, 22 (1964), p. 128-157.

Green, Judith A., The Government of England under Henry I, Cambridge, Cambridge University Press, 1986.

Hamilton, Bernard, "The Growth of the Latin Church of Antioch and the Recruitment of its Clergy", in East and West in the Medieval Eastern Mediterranean. Antioch from the Byzantine Reconquest until the End of the Crusader Principality, éd. K. Ciggaar et M. Metcalf, Leuven - Paris - Dudley, Uitgeverij Peeters en Department Oosterse Studies, 2006, p. 171-183.

Hamilton, Bernard, The Latin Church in the Crusader States. The Secular Church, Londres, Variorum, 1980.

Hiestand, Rudolf, «Ein unbekanntes Privileg Fürst Bohemonds II von Antiochia für das Hospital vom Marz 1127 und die Frühgeschichte der antiochenischen Fürstenkanzlei», Archiv für Diplomatik, 43 (1997), p. 27-46. 
Hiestand, Rudolf, «Antiochia, Sizilien und das Reich am Ende des 12. Jahrhunderts", in Quellen und Forschungen aus Italienischen Archiven und Bibliotheken, 73 (1993), p. 70-121.

Hill, John Hugh, Hill, Laurita Lyttleton, Raymond IV de Saint-Gilles. 1041 (ou 1042)1105, Toulouse, Privat, 1959.

Hillenbrand, Carole, «The career of Najm al-Dīn İl-Ghāzī», Der Islam, 58 (1981), p. 250-292.

Holt, Peter Malcolm, The Crusaders States and their Neighbours, 1098-1291, Londres, Longman, 2004.

Houben, Hubert, I Normanni, Bologne, Il Mulino, 2013.

Huscroft, Richard, The Norman Conquest. A New Introduction, Harlow, PearsonLongman, 2009.

Irwin, Robert, «Usamah ibn Munqidh: An Arab-Syrian Gentleman at the Time of the Crusades Reconsidered", in The Crusades and their Sources. Essays Presented to Bernard Hamilton, éd. J. France et W.G. ZaJAC, Aldershot, Ashgate, 1998, p. 71-87.

Jamison, Evelyn, «Some Notes on the "Anonymi Gesta Francorum", with Special Reference to the Norman Contingent from South Italy and Sicily in the First Crusade», in Studies in French Language and Medieval Literature. Presented to Professor Mildred K. Pope, Manchester, Manchester University Press, 1939, p. $183-208$.

Lapina, Elizabeth, Warfare and the Miraculous in the Chronicles of the First Crusade, University Park, Pennsylvania University Press, 2015.

Ligato, Giuseppe, «Le vicende della crociata lombarda. Gerusalemme o "regnum Babilonicum"?», in Deus non voluit. I Lombardi alla prima crociata (1100-1101). Dal mito alla ricostruzione della realtà, éd. G. Andenna et R. Salvarani, Milan, Vita e Pensiero, 2003, p. 31-103.

LoRenz, Stefan, Bohemund von Tarent und die Normannen als Herrscher von Antiochen, Norderstedt, Grin, 2007.

Loud, Graham A., «The "Gens Normannorum”. Myth or Reality?», in Anglo-Norman Studies, IV, éd. R.A. Brown, Woodbridge, Boydell, 1982, p. 104-116.

Loud, Graham A., "Norman Italy and the Holy Land», in Horns of Hattin, éd. B.Z. KeDAR, Jerusalem, Variorum, 1992, p. 49-62.

Loud, Graham A., "Il regno normanno-svevo visto dal regno d'Inghilterra», in Il Mezzogiorno normanno-svevo e le Crociate, éd. G. MuscA, (Atti delle quattordicesime giornate normanno-sveve), Bari, Dedalo, 2002, p. 175-195.

MacEviti, Christopher, The Crusades and the Christian World of the East: Rough Tolerance, Philadelphia, University of Pennsylvania Press, 2008.

Mallett, Alex, "The "Other" in the Crusading Period: Walter the Chancellor's Presentation of Najm al-Dīn Il-Ghāzī», Al-Masaq: Islam and the Medieval Mediterranean, 22 / 2 (2010), p. 113-128.

MARTin, Jean-Marie, «Les structures féodales normanno-souabes et la Terre Sainte», in Il Mezzogiorno normanno-svevo e le Crociate, éd. G. Musca, (Atti delle quattordicesime giornate normanno-sveve), Bari, Dedalo, 2002, p. 225-250.

Matthew, Donald J.A., L'Europa normanna, (trad. it.), Rome, Jouvence, 1987. 
Mayer, Hans Eberhard, «Jérusalem et Antioche au temps de Baudouin II», Comptes rendus des séances de l'Académie des Inscriptions et Belles-Lettres, 124/ 4 (1980), p. $717-734$.

Mayer, Hans Eberhard, Varia Antiochena: Studien zum Kreuzfahrerfürstentum Antiochia im 12. und frühen 13. Jahrhundert, (Monumenta Germaniae Historicae Studien und Texte, 6), Hannovre, Hahn, 1993.

Murray, Alan V., «How Norman was the Principality of Antioch? Prolegomena to a Study of the Origins of the Nobility of a Crusader State», in Family Trees and the Roots of Politics: The Prosopography of Britain and France from the Tenth to the Twelfth Century, éd. K.S.B. Keats-Rohan, Woodbridge, Boydell \& Brewer, 1997, p. 349-359.

Musarra, Antonio, In partibus Ultramaris. I genovesi, la crociata e la Terrasanta (secc. XII-XIII), Rome, ISIME, à paraître.

Mutafian, Claude, "L'enjeu cilicien et les prétentions normandes (1097-1137)", in Autour de la Première croisade (Byzantina Sorbonensia 14), éd. M. BALARD, Paris, Publications de la Sorbonne, 1996, p. 453-461.

Norman Expansion. Connections, Continuities and Contrasts, éd. K.J. STRINGER et A. Joтischкy, Farnham, Ashgate, 2013.

Oıкоnomides, Nicolas, «The Usual Lead Seal», in Dumbarton Oaks Paper, 27 (1983), p. 147-157.

Papadakis, Aristeides, Meyendorff, Jean, The Christian East and the Rise of the Papacy: the Church 1071-1453 A.D., (Church History, 4), Crestwood (NY), St. Vladimir's Seminary Press, 1994.

Phillips, Jonathen, Sacri guerrieri. La straordinaria storia delle Crociate, (trad. it.), Rome - Bari, Laterza, 2011 [éd. orig. New York 2009].

Records, Administration and Aristocratic Society in the Anglo-Norman Realm, éd. N. VinCEnT, Woodbridge, Boydell \& Brewer, 2009.

Rey, Emmanuel Guillaume, «Résumé chronologique de l'histoire des princes d'Antioche», Revue de l'Orient latin, IV (1896), p. 321-407.

REy, Emmanuel Guillaume, «Les dignitaires de la principauté d'Antioche. Grandsofficiers et patriarches (XI'-XIII ${ }^{e}$ siècle) », Revue de l'Orient latin, VIII (1900-1901), p. 116-157.

Riley-Smith, Jonathan, The First Crusaders, 1095-1131, Cambridge, Cambridge University Press, 1997.

Rowe, John G., «Paschal II and the Relation between the Spiritual and Temporal Powers in the Kingdom of Jerusalem», in Speculum, XXXII (1957), p. 470-501.

Russo, Luigi, Boemondo. Figlio del Guiscardo e principe di Antiochia, Avellino, Elio Sellino, 2009.

Russo, Luigi, «Il Liber di Raimondo d'Aguilers e la Sacra Lancia d'Antiochia», Studi Medievali, XLVII, fasc. II (2006), p. 785-837.

Russo, Luigi, «Il Mediterraneo agli occhi di un pellegrino della "prima crociata" ", in Studi in onore di Guglielmo de Giovanni-Centelles, éd E. Cuozzo, Salerne, SISAUS, 2010, p. 113-123. 
Russo, Luigi, "I Normanni e il movimento crociato. Una revisione», in Il Papato e $i$ Normanni. Temporale e Spirituale in età normanna, a cura di E. D'ANGELo, C. LeONARDI, Florence, SISMEL, 2011, p. 163-174. [réimprimé dans ID., I Normanni del Mezzogiorno e il movimento crociato, Bari, Mario Adda, 2014, p. 23-36].

Russo, Luigi, «Il regno normanno del Mezzogiorno e “Outremer”: Scambi, interazioni e mancate occasioni", in Gli Italiani e la Terrasanta, éd. A. Musarra, Florence, SISMEL, 2014, p. 137-149 [réimprimé dans ID., I Normanni del Mezzogiorno e il movimento crociato, Bari, Mario Adda, 2014, p. 143-154].

Russo, Luigi, «Tancredi e i bizantini. Sui “Gesta Tancredi in expeditione Hierosolymitana” di Rodolfo di Caen", in Medioevo Greco, 2 (2002), p. 193-230.

Russo, Luigi, «Il viaggio di Boemondo d'Altavilla in Francia (1106) : un riesame», in Archivio Storico Italiano, 163 (2005), p. 3-42 [réimprimé dans ID., I Normanni del Mezzogiorno e il movimento crociato, Bari, Mario Adda, 2014, p. 101-142].

SANsone, Salvatore, Tra cartografia politica e immaginario figurativo. Matthew Paris e l'Iter de Londinio in Terram Sanctam, Rome, ISIME, 2009 (Nuovi Studi Storici 84).

SHEPARD, Jonathan, «Imperial Troubleshooters: Cultural Representatives and Master of Improvisation", in Ambassadeurs et ambassades au coeur des relations diplomatiques. Rome, Occident médiéval, Byzance (VIII ${ }^{e}$ s. avant JC-XII ${ }^{e}$ s. après JC), éd. A. BECKER et N. Drocourt, Metz, Centre de recherche universitaire Lorrain d'histoire, 2012, p. 351-369.

Talbert, Richard J.A., Rome's World. The Peutinger Map Reconsidered, Cambridge, Cambridge University Press, 2010.

Thомаs, Hugh, The Norman Conquest. England after William the Conqueror, Lanham, Rowman \& Littlefield, 2008.

Tyerman, Christopher, Le guerre di Dio. Nuova storia delle crociate, trad. italiana, Turin, Einaudi, 2012 [éd. orig. Cambridge 2006].

Vorderstrasse, Tasha, "Trade and Textiles from Medieval Antioch», Al-Masaq: Islam and the Medieval Mediterranean, 22/ 2 (2010), p. 151-171. 